\title{
SOLACE: A Framework for Electronic Negotiations
}

\author{
Oyindamola Abass and Gheorghita Ghinea \\ School of Information Systems, Computing and Mathematics \\ Brunel University, Uxbridge, Middlesex. UB8 3PH, UK
}

\begin{abstract}
Most existing frameworks for electronic negotiations today are tied to specific negotiation systems for which they were developed, preventing them from being applied to other negotiation scenarios. Thus, the evaluation of electronic negotiation systems is difficult as each one is based on a different framework. Additionally, each developer has to design a new framework for any system to be developed, leading to a 'reinvention of the wheel'. This paper presents SOLACE - a generic framework for multi-issue negotiations, which can be applied to a variety of negotiation scenarios. In contrast with other frameworks for electronic negotiations, SOLACE supports hybrid systems in which the negotiation participants can be humans, agents or a combination of the two. By recognizing the importance of strategies in negotiations and incorporating a time attribute in negotiation proposals, SOLACE enhances existing approaches and provides a foundation for the flexible electronic negotiation systems of the future.
\end{abstract}

\section{KEYWORDS}

electronic negotiation, frameworks, multi-attribute negotiation

Reprint requests to: Oyindamola Abass, e-mail: oyindamola.abass, george.ghinea@ brunel.ac.uk 


\section{INTRODUCTION}

Negotiation is an essential part of commerce. From the days of the barter system to present day electronic commerce, negotiations have taken place to ensure some degree of fairness and accountability. Negotiations exist in other aspects of our lives as well - they appear in a multitude of forms, they take place in many different situations, and they are influenced by ethical, cultural, and social circumstances. The variety and diversity of roles of negotiators and negotiation situations challenge researchers from many disciplines, including Anthropology, Psychology and Sociology, Political Sciences, Economics, Law, and Applied Mathematics (Kersten, 2003).

Electronic commerce information systems operate in domains that differ with respect to negotiation. Research into electronic negotiations has provided a variety of fully automated and semi-automated negotiation systems employing the use of agents, decision support systems, and negotiation support systems (Zlatev \& Van Eck, 2003). Nevertheless, the general frameworks for automated negotiation that have been proposed in the past few years (Wong et al., 2000, Jennings et al., 2001; Bartolini et al., 2002) do not pay attention to these differences, making it difficult to assess which is the most applicable in a specific negotiation domain. Most negotiation systems are based on different frameworks and models, with each researcher developing a framework for their own system. A few attempts (Strobel, 2001; Bartolini et al., 2002) have been made at developing generic frameworks or models, but more research still has to be carried out in developing electronic negotiation frameworks to create more robust and standardized frameworks. This approach will prove very beneficial to all parties in this field of research by providing a standard on which future works in electronic negotiation support can be based.

The aim of our work is to develop a generic electronic negotiation framework-SOLACE - that will serve as a reference point for electronic negotiation systems. This framework will provide a common platform on which all developed systems can be evaluated and reduce development time as there would be no need to 're-invent the wheel' by creating new frameworks when developing negotiation systems. SOLACE will address such current issues facing electronic negotiations as multi-attribute negotiation, 
negotiation strategies, and hybrid-system support. In our work, we have adopted a quantitative positivist research method and demonstrated the practical utility and feasibility of our framework through a proof-of-concept application.

The rest of this paper is structured as follows: Section 2 gives a background on electronic negotiation frameworks. Section 3 introduces SOLACE. An example of electronic negotiations under the SOLACE framework is described in Sec. 4, and an evaluation of the functionality of SOLACE is given in Sec. 5. Lastly, conclusions are drawn in Sec. 6.

\section{NEGOTIATION FRAMEWORKS}

Negotiation is a process of social interaction and communication that involves distribution and redistribution of power, scarce resources, and commitments. The interpersonal character, the participants' independence as the decision-making entities, and their interdependence in their inability to achieve goals unilaterally contribute to the negotiation complexity (Kersten, 2003). Electronic negotiations occur whenever the interactions of the negotiating parties are transmitted by means of electronic media. Such negotiation processes are conducted fully or partially with the use of software. Research into electronic negotiations has mainly focused on implementing negotiation systems but has neglected the modeling aspects of negotiations. Hence, unique and proprietary solutions are created repeatedly, with enormous efforts spent on integrating isolated solutions. The development of generic electronic negotiation frameworks can solve this problem. Before we proceed further, however, we must understand what we mean by frameworks.

\subsection{What are Frameworks?}

A framework is a reusable design expressed as a set of abstract classes. A framework is a reusable design for all or part of software. By its very definition, a framework is an object-oriented design. Although, a framework doesn't have to be implemented in an object-oriented language, it usually is (Johnson, 2004). Frameworks are often described as patterns. Yet, even 
though the two are in some ways similar, the main difference between them is that whereas the pattern tells the reader how to solve a problem, the framework usually provides a canned solution (Visual Works, 2004).

Frameworks are important for the efficiency of negotiation processes, for flexibility-bringing about customization and creating a scenario for the success of resulting settlements. In electronic negotiations, modeling aspects have been neglected, with the undesirable consequence that discussing agreement scenarios on a conceptual level is difficult, and that design efforts cannot be reused and refined in the implementation phase in a formal way (Strobel, 2001). Thus, a need exists for general models of negotiations, which could be used to characterize the nature and process of negotiations, formalize its aspects, and which have a flexibility to describe a wide range of possible structures and interactions.

\subsection{Existing Frameworks}

Recent software frameworks for the design and implementation of e-negotiation media and support tools include SMACE (Cardoso \& Oliveira, 2000), DynamiCS (Tu et al., 2001), INSULA (Benyoucef et al., 2001), SILKROAD (Strobel, 2001), MAP (Bichler et al., 2002), and Bartolini et al., 2002. In SILKROAD, Strobel proposed a design and application framework for electronic negotiations. Based on this framework, organizations creating an electronic market or sellers intending to offer potential buyers the option to bargain can generate, in a flexible and efficient way, customized electronic negotiation systems supporting the roles and protocols designed. SILKROAD also addresses the notion that today's agreement services, such as electronic auctions or agent systems, are flexible or configurable enough to support the requirements of a larger range of existing or emerging agreement scenarios in business practice (Strobel, 2001).

Another group of researchers who have put forward an e-negotiation framework is Bartolini et al. (2002). Accordingly, these authors designed a generic interaction protocol and a general interaction framework using the same protocol, which can be parameterized with different negotiations. Depending on the choice of rules, different negotiation mechanisms can be 
implemented. The authors argue that this design is more flexible in that only the general interaction framework has to be agreed in advance; rules can be defined at any time. The other advantage is that protocol specifications can be explicitly passed between agents and reasoned over.

Tu et al. (2001), on the other hand, developed DynamiCS - an actorbased framework for negotiating mobile agents. This framework is based on the notion of an actor system, which decomposes an application component into autonomously executing subcomponents cooperating with each other. The framework is based on a plug-in mechanism enabling a dynamic composition of negotiating agents. The architecture puts a strong emphasis on the notion that mobility and intelligence are not opposed, but rather orthogonal to one another.

Lee (2000) also proposed a framework, which emphasizes the time involved in a negotiation process. Lee proposes that a time attribute be attached to each message to represent the period of time in which the message is valid. This framework assumes that a system and protocol for message exchange already exist, thus they can be adopted only in conjunction with other frameworks.

Related is the work of Cardoso and Oliveira (2000), who presented SMACE, a layered platform for agent-mediated electronic commerce, supporting multilateral and multi-issue automated negotiations. SMACE is a multi-agent system for electronic commerce, for which users can create buyer and seller agents that negotiate autonomously to reach agreements about product transactions. In this system, the negotiation infrastructure through which the software agents interact is independent of their negotiation strategies.

Jennings et al. (2001) developed a generic framework for classifying and viewing automated negotiations. This framework was subsequently used to discuss and analyze the three main methods of approach that have been adapted to automated negotiation, namely, game-theoretic, heuristic, and argumentation-based approaches. The authors concluded that the gametheoretic approach fails to generate a general model governing rational choice in interdependent situations. Instead, the discipline has produced a number of highly specialized models that are applicable to specific types of interdependent decision-making. On the other hand, the heuristic approaches 
aim to produce good rather than optimal solutions. The authors suggest the use of argumentation-based approaches to allow the exchange of additional information and to make it possible for agents to handle conflicting information.

In contrast, Wong et al. (2000) proposed a framework based on CaseBased Reasoning (CBR). Case-Based Reasoning is an approach to use past experience for choosing concrete strategy in every situation. The framework is built on top of a database with information on past negotiations. This approach, although very useful, requires a lot of information gathering and might be influenced by some historical factors present at the time the prior negotiations occurred, but which may have become irrelevant in the present scenario.

Bichler et al. (2002) developed The Multidimensional Auction Platform (MAP) as a set of software modules for building multidimensional auction markets. The MAP is an extensible object framework that enables the reuse of the advanced allocation algorithms as a standard solver component in electronic markets. This framework provides a declarative interface and sheds developers from the complexities of a particular allocation algorithm. Lastly, Bellosta et al. (2004) developed a multi-criteria model for electronic auctions, which is based on reference points. This model allows the buyer agent to control the negotiation process on each attribute of the deal.

Although this enumeration is not exhaustive, it does illustrate the breadth of approaches. As can be seen above, most existing frameworks (Cardoso \& Oliveira, 2000; Tu et al., 2001; Bichler et al., 2002) are suited to the particular needs of the system at hand and cannot be applied across the board in other scenarios. On the other hand, the frameworks by Strobel, (2001) and Bartolini et al., (2002) fulfill many functions of an e-negotiation platform. These authors describe the negotiation protocol in detail and it can be applied to ranging scenarios. Nonetheless, these frameworks have their shortcomings as well, in terms of complexity, ignorance of the time attribute, and negotiation strategies.

Here we present the development of a comprehensive, generic framework-SOLACE, which deals with multi-issue negotiations and recognizes negotiation strategies as an important aspect of the negotiation process. 


\section{SOLACE-AN ELECTRONIC NEGOTIATION FRAMEWORK}

\subsection{Design Philosophy}

SOLACE combines concepts from existing frameworks, as well as some novel ideas to produce an efficient, multi-issue negotiation framework. The strongest points of SOLACE are in allowing multi-attribute negotiation as well as its simplicity and flexibility, together with its incorporation of the human angle and inclusion of the finite time guarantee strategy. These characteristics will enable developers to adopt it easily for building electronic negotiation systems in the future and will help researchers in evaluating and comparing systems, which was very difficult in the past as the systems were based on different frameworks.

\subsection{Components of the Framework}

The major components of SOLACE are negotiation protocols, negotiation objects, negotiation strategy, negotiation host, and negotiation participants.

Negotiation participants (agents or humans) interact with the entire system and their involvement varies for different scenarios. The negotiation strategy is very important in reaching an agreement in a negotiation process with the particular strategy adopted possibly determining what issues will be negotiated. The bi-directional arrow in Fig. 1 indicates that the strategy may also be determined by the particular negotiation scenario, i.e. what is being negotiated and/or the parties involved. Participants can negotiate on a variety of objects (e.g. price, quality) through interaction of the protocol. The strategy adopted influences all aspects of the system. The negotiation host coordinates all the activities in the system.

Participants in the negotiation space can bargain on several issues depending on the particular scenario. For example, in the buying and selling of goods, the issues could be price, delivery date, and quality. The negotiation of these issues is done simultaneously, and the three issues together determine the type of agreement reached. The protocol is responsible for all messagepassing. The framework also imposes a finite time guarantee characteristic on 
Fig. 1: SOLACE - A framework for electronic negotiations

the protocol ${ }^{1}$ and is incorporated at this stage. All proposals made by both parties are accompanied with a validity period after which the proposing party may reject agreements to that proposal.

A typical negotiation occurs with participants negotiating with each other on issues via the protocol based on their strategies in order to reach an agreement. The host facilitates this process. Each agent can communicate with more than one agent, which in turn can be dealing with several other agents. The components shall now be described in detail.

3.2.1 Negotiation protocols. A negotiation protocol is the set of rules that govern the interaction between entities in an e-commerce transaction. This set includes rules governing the types of participants, the roles such participants can play, the possible negotiation states, and the events that can trigger such transitions to these states.

Table 1 shows properties from Jennings et al. (2001) that have been adapted into this framework as characteristics that must be met by the protocol at the heart of the SOLACE framework:

\footnotetext{
${ }^{1}$ Adopted from Lee (2000) in which each offer made has a timeline associated with it, after which it expires.
} 
TABLE 1

Properties of the SOLACE negotiation protocol

\begin{tabular}{|l|l|}
\hline Property & $\begin{array}{l}\text { Meaning and Application in SOLACE } \\
\text { Stability }\end{array}$ \\
\hline Simplicity & $\begin{array}{l}\text { It must provide all agents the incentive to behave in the } \\
\text { party and strictly adheres to the agents' strategies. }\end{array}$ \\
\hline Distribution & $\begin{array}{l}\text { It is easy to understand and follow. The rules of negotiation } \\
\text { are broken down into the simplest if-then-else statements } \\
\text { making the logic very easy to understand. }\end{array}$ \\
\hline $\begin{array}{l}\text { The protocol must be designed in such a way that there is } \\
\text { no single point of failure. The protocol can carry out } \\
\text { negotiation with several parties simultaneously, thus there } \\
\text { is no single point of failure. Also, in order to minimize } \\
\text { communication overhead, the least amount of proposals } \\
\text { required to reach an agreement is made. }\end{array}$ \\
\hline Guaranteed Success & $\begin{array}{l}\text { The protocol must guarantee success, in that an agreement } \\
\text { must be reached or a decision to terminate, which is agreed } \\
\text { to by both parties. This is achieved by ensuring both parties } \\
\text { agree to decisions made, either to conclude a contact or to } \\
\text { terminate a negotiation process }\end{array}$ \\
\hline Individual & $\begin{array}{l}\text { The protocol must generate Pareto efficient negotiations. } \\
\text { The strategies employed by the agents are unknown to each } \\
\text { other. }\end{array}$ \\
\hline Rationality & $\begin{array}{l}\text { The protocol must be individually rational as incentive for } \\
\text { the agents. A protocol is said to be rational if playing by } \\
\text { the rules is in the best interests of all parties. The protocol } \\
\text { is not biased towards either party }\end{array}$ \\
\hline
\end{tabular}

3.2.2 Negotiation objects. Negotiation objects are the range of issues over which agreement must be reached. These objects are commonly referred to as issues, e.g. price, delivery date, and quality. SOLACE allows the negotiation of several objects simultaneously with several agents.

SOLACE supports both static and dynamic objects by allowing either party to introduce other issues apart from those being negotiated. For

\footnotetext{
${ }^{2}$ A negotiation is said to be Pareto efficient if there is no other outcome that will
} make at least one agent more efficient without making any other agent worse off 
example, a seller can throw in a $10 \%$ discount on a next purchase to get the buyer enticed. This introduces 'discount' as a new object of negotiation and the buyer can ask for a higher discount say $20 \%$, increasing the number of objects for bargaining by one (dynamic objects).

3.2.3 Negotiation strategy. The negotiation strategy drives the entire negotiation process. The inclusion of strategies in SOLACE is at a conceptual level, and shows how strategies can determine the interaction between its constituent components.

Although some researchers have argued against this approach, saying that negotiation strategies lead to complete automation of electronic negotiations (Strobel, 2001) or indeed that the strategies will either be too simple (easily deciphered) or too complex to be formalized or that the strategies will not gain the trust of users (Beam et al. 1996), the importance of strategies cannot be over-emphasized, as strategies distinguish the winners and losers in any scenario.

Negotiation strategies are usually inspired from game theory and heuristics. The game theory approach is based on rational behavior and common knowledge, whereas the heuristic approach is based on imitating human behavior. Generally, the agents strive to achieve a collaborative scenario in which all parties in a negotiation process could generate offers that satisfy their wishes (Rahwan et al., 2004). SOLACE, while stressing the importance of strategy in negotiations, leaves the adoption of the specific strategies to the discretion of the developer of the negotiation system.

3.2.4 Negotiation participants. Negotiation participants are the agents or users that negotiate using the system. One of the major characteristics of SOLACE is its user involvement. Many of the existing frameworks assume full automation, ignoring the human angle. Systems built using this framework will be hybrid systems allowing users to specify their level of involvement. The next section discusses negotiation in SOLACE.

\section{NEGOTIATION IN SOLACE: A PROOF OF CONCEPT APPLICATION}

This section provides the systems analysis and design of a proof of concept application based on the SOLACE framework. A typical negotiation 
scenario at an Estate Agency is discussed. The systems analysis and design of the Property Negotiation System (PNS) is thus carried out.

\subsection{Scenario}

Alpha Properties Ltd is an Estate Agency. They are currently in the process of re-engineering their business processes and they would like a system to assist them in this process. The proposed system will negotiate on behalf of buyers and sellers. It is hoped that this system will allow agreements to be reached faster, efficiently and without any human bias.

\subsection{Working with the Framework}

The SOLACE framework will be used to develop the PNS. The elements of the framework are discussed below with respect to the proposed system.

\section{Negotiation Participants}

The Negotiation participants are the users and the agents. Users are potential buyers or sellers of property.

\section{Negotiation Objects}

The Negotiation objects include price, number of bedrooms, number of parking places, type of house, the age of the house, location, fully fitted kitchen, etc.

\section{Negotiation Strategy}

The buyer agent has a strategy of looking for the lowest priced property, which has all the other specified criteria present. The seller agent has a strategy of looking for the highest priced bid for the property it has available. Negotiating users adopt whatever strategy they choose and may even change their strategy several times during the negotiation process. Upon examination of the agents' strategies, it appears as though they are distributive strategies with each agent striving to achieve its objective at the other's expense. However, if we consider that the two 
agents have complementary objectives - buying and selling, the strategies can be said to be integrative.

\section{Negotiation Protocol}

The Negotiation protocol contains the negotiation rules for the system. Rules drive the activity in the system by describing the actions to take place when a specified set of conditions is met.

The following rules are defined for the Negotiation protocol.

- Users must be registered in the system before they can negotiate

- Property registration triggers the negotiation process

- Agents can bargain with more than one agent at a time

- Agreements are formed with the consent of two parties

\subsection{A PNS Prototype under SOLACE}

We have implemented a proof of concept PNS prototype under the SOLACE framework, using a combination of Java and XML technologies. Figure 2 is a representation of the System architecture of the PNS, whereas Fig. 3 illustrates a typical negotiation process. The input comes from data contained in XML documents and the user interface, which is accessed through the JDOM API and then processed by the Java application engine.

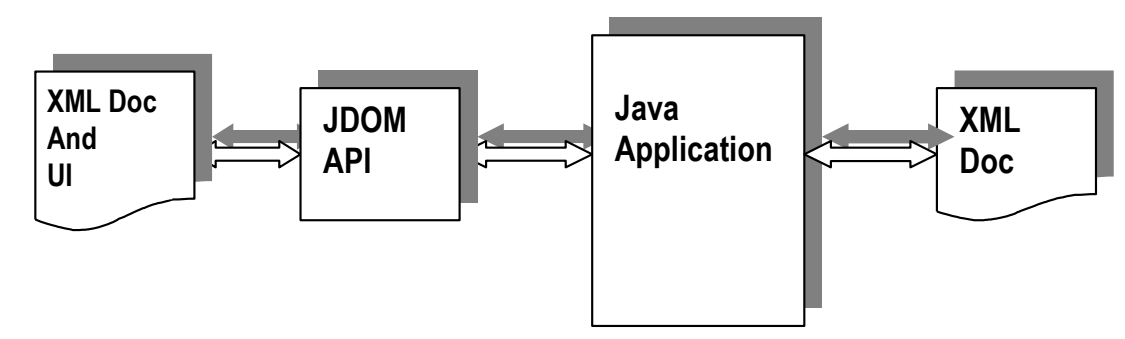

Fig. 2: System architecture for the property negotiation system for alpha properties 


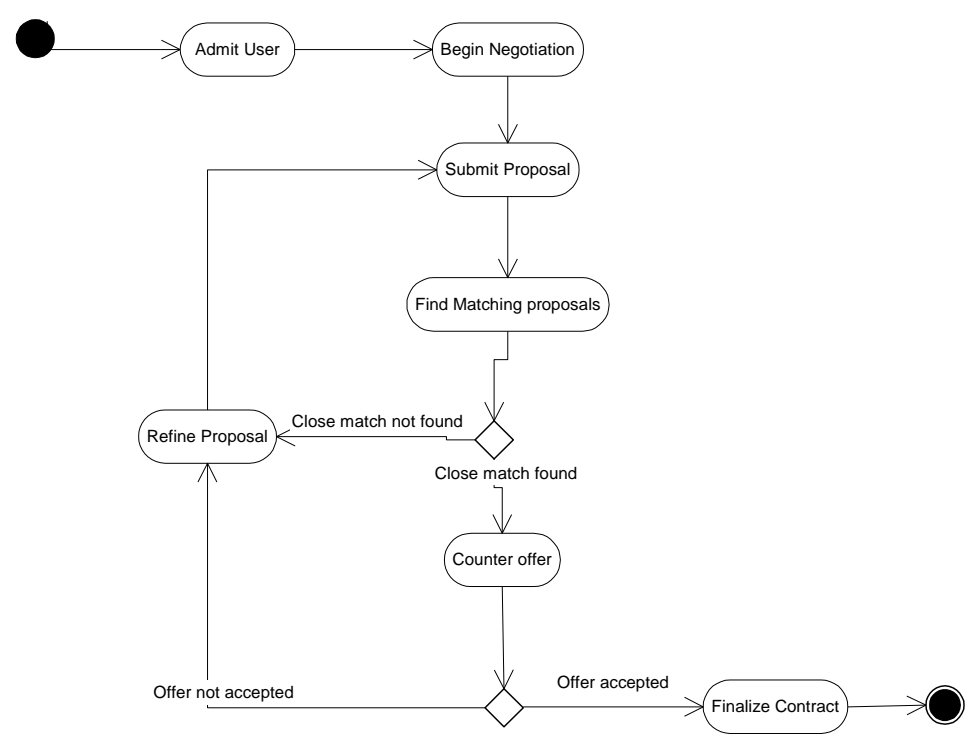

Fig. 3: Activity diagram showing the negotiation process

The output is then stored as XML documents JDOM, and with all its useful features has some drawbacks. As at the time of implementing, JDOM did not have robust XML Schema Definition (XSD) support, but Document Type Definitions (DTDs) were supported extensively. The richer and more extensible nature of XSDs and their support for namespaces and data types would have been preferred for this implementation. Nevertheless, DTDs will provide us with the basic functionality we need at this time.

\subsection{PNS System Requirements}

From an analysis of the business case, the following requirements have been identified for the system.

- Users should be able to query the system based on specified criteria.

- The system should be able to negotiate on behalf of potential buyers.

- The system should be able to negotiate on behalf of potential sellers.

- The system should bring about agreements between potential buyers and sellers.

- The system should document all agreements between parties. 


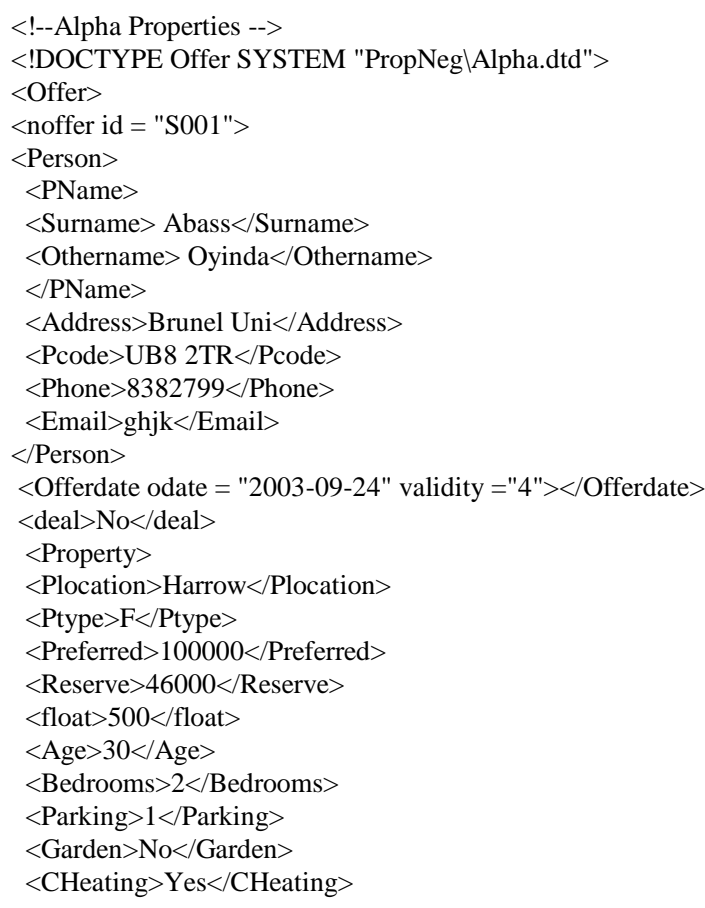

Fig. 4: XML representation of electronic negotiation structure under SOLACE

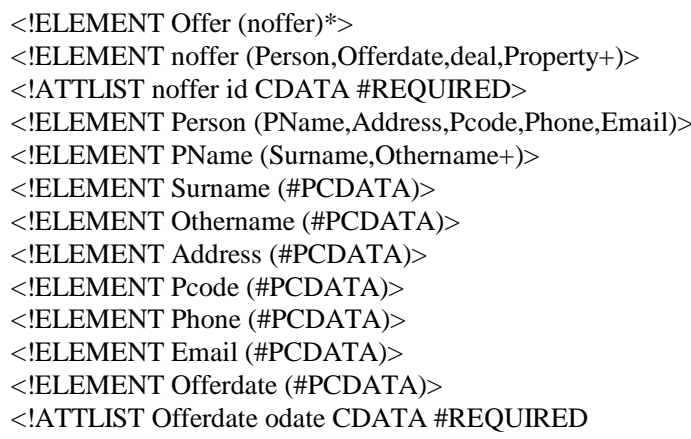

Fig. 5: DTD representation of electronic negotiation structure under SOLACE 


\subsection{Requirements Representation in XML}

The prototypical implementation of the PNS uses XML documents (Fig. 4) to represent the system requirements of the PNS, with the underlying structure being specified by a DTD (Fig. 5). In XML documents, the elements are the building blocks, with attributes providing additional information about the elements. We give below some element and attribute descriptions:

- Offer - This is the root element of the document. Every XML document should contain a single root element. The Offer element consists of one or more noffer (new offer) elements. Each potential buyer or seller has his details specified as an 'noffer' in their respective XML documents.

- Noffer - Each noffer has four child elements, namely: Person, Offerdate deal, and Property (described below). A new offer can contain requests or offers of more than one property, but it can have only one Person, Offerdate, and deal element associated with it.

- Person - The Person element stores personal details of the buyer or the seller, including name, address, and phone number.

- Property - The property element stores details of the house on offer (in the case of a seller) or the house requested (in the case of the buyer). The Property element contains nine child elements, which capture specific details about the property such as type and location of property, number of bedrooms, etc. In addition, it contains the Preferred element, which stores the users preferred buying, or selling price; the Reserve element, which contains the lowest price for which, the property can be sold (seller), or the highest price it can be bought (buyer). It also contains the Float element, which stores the initial increment or decrement value during the negotiation process.

\subsection{Implementation of Framework Requirements}

This section describes the requirements of the system as imposed by the framework. Any system implemented should satisfy the main characteristics of electronic negotiation systems as defined by SOLACE: 
Multi-Issue Negotiation. The PNS allows users to bargain on several issues, such as price, number of bedrooms, parking places, etc. The issues to be negotiated are dynamic. The seller agent introduces an offer of increasing the number of bedrooms and parking places after a prescribed number of proposals without an agreement.

Human Interaction/Hybrid System. SOLACE prescribes that the system should involve user interaction at the agreement stage or should conclude negotiations on their behalf. Our PNS implements this functionality by allowing the user to accept or deny offers made or allowing agents to conclude transactions based on previously specified terms. For example, the agent can accept an offer on the buyer's behalf if the offer falls below his reserve price; if, however, the offer is above his reserve price, then the system prompts the user to accept or reject the offer.

Time/Validity. The time attribute is associated with each offer made by either party. At the initial stage, the parties specify the validity of the offers made. The intelligent buyer agent varies its validity period, depending on the sequence of negotiation proposals. The seller agent, on the other hand, simply has a basic plan that decreases the validity after a prescribed number of proposals.

Platform Independence. Java and XML are platform independent, thus satisfying this criterion for the implementation of the PNS as prescribed by SOLACE.

Negotiation Strategies. The two agents implemented have different strategies. As the buyer agent satisfies certain compulsory characteristics of intelligent agents - autonomy and ability to learn — we can say that the buyer is intelligent. The ability to learn is achieved by adapting its offers in response to the seller's offers. The buyer agent's autonomy stems from the notion that it is capable of acting independent of the user.

The seller agent's strategy is based on the number of proposals made so far. If after a prescribed amount of proposals an agreement has not been reached, then the seller amends his offers. The strategy here is not 
intelligent (no learning involved), the agent reacts in the same way, irrespective of the buyer's offers. The strategies implemented though simple do illustrate the importance of strategies in negotiations.

\author{
Buyer Agent \\ If the difference in seller's offer has been increasing \\ Seller getting aggressive \\ Maintain steady decrease in offer price \\ Else if the difference in sellers' offers has been the same or decreasing \\ Seller loosing interest \\ Become aggressive by increasing offers to keep the seller's interest \\ Else prompt seller to modify his offer \\ Seller Agent \\ Maintain a steady decrease in offer price \\ If number of proposals equals $n$ \\ Become aggressive by decreasing offer price geometrically \\ If number of proposals equals $n+m$ \\ Revert to steady decrease in offer price \\ The values of $\mathrm{n}$ and $\mathrm{m}$ can be supplied by the user or the developer
}

Fig. 6: Pseudocode for agents' strategies

\title{
5. EVALUATION OF FRAMEWORK REQUIREMENTS
}

This section contains the evaluation of the requirements imposed on the system by SOLACE.

\subsection{Multi Issue Negotiation}

Multi issue negotiation allows the user to negotiate on more than one issue simultaneously. The figure below shows a negotiation process where the number of bedrooms and parking places are introduced as new issues for bargaining in addition to the price already being negotiated hence depicting dynamic multi-attribute negotiation. 


\section{Ualidity :4hours}

The no of bedrooms will be increased to : 3

Do you Accept the of fer $\mathbf{Y} / \mathrm{N}$ ?

n

Buyer - Proposed Price : 35102.0

Ualidity: 4 hours

The no of bedrooms will be increased to :37

The no of parking places will be increased to :2

Seller - New offer Price =71500.0

Ualidity : 4 hours

Do you Accept the Offer $Y / N$ ?

n

Buyer - Proposed Price : 35403.0

Ualidity: 4 hours

The no of bedrooms will be increased to :3

The no of parking places will be increased to :2

Seller - New Offer Price =67000.0

Ualidity : 4hours

Do you Afccept the Offer $Y / N$ ?

n

Buyer - Proposed Price : 36393.0

Ualidity: 4 hours

The no of bedrooms will be increased to :3

The no of parking places will be increased to : 2

Seller - New offer Price $=64509.9$

Fig. 7a: Multi issue negotiation with dynamic objects

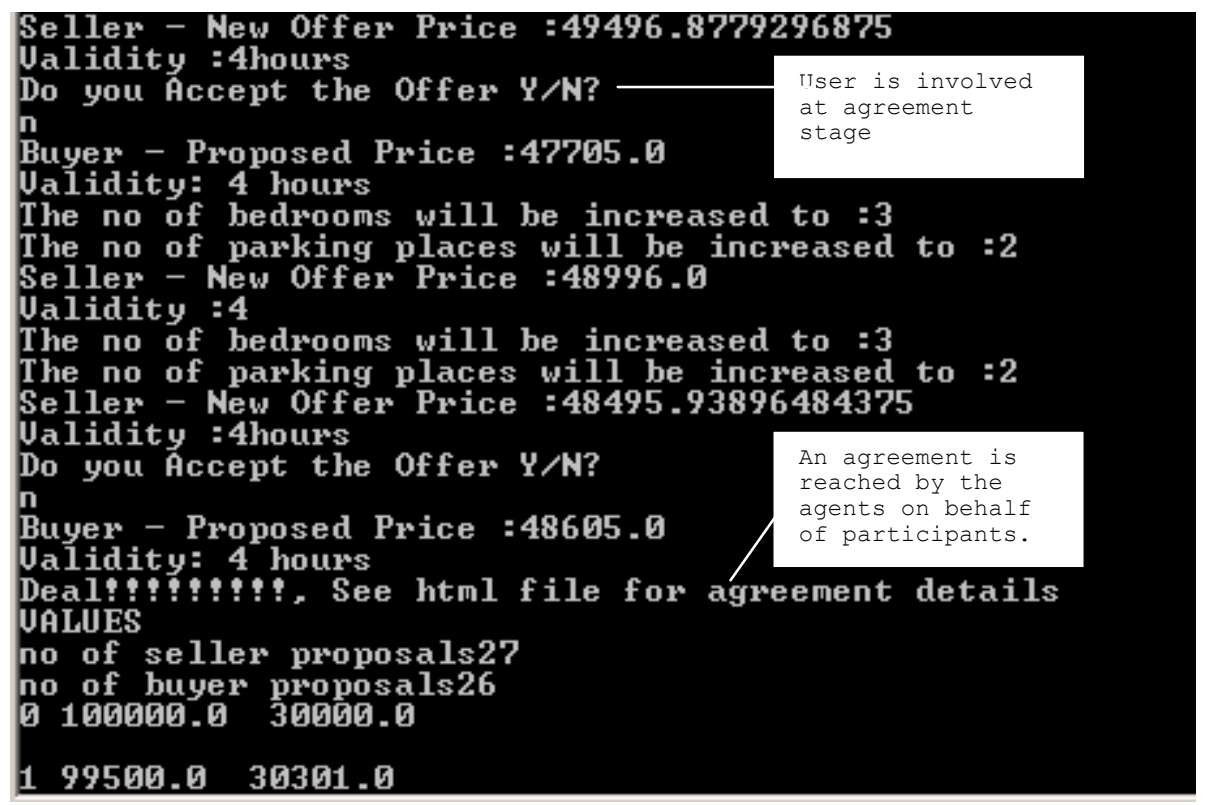

Fig. 7b: Hybrid system functionality 


\subsection{Human Interaction/Hybrid System}

We depict in Fig. 7b above highlights of a scenario showing the hybrid capabilities of the system. The first scenario shows an agent concluding an agreement on behalf of the user based on previously specified criteria.

\subsection{Negotiation Strategy}

As described in the previous section, the negotiation strategies of the agents differ with the buyer agent being more intelligent. The graph below shows the sequence of proposals made by the buyer and the seller in a negotiation scenario. The graph shows the buyer agent responding appropriately to changes in the seller's offer.

From the graph shown in Fig. 7c, one can observe that the seller agent maintains a steady decrease in his offer price until the fourth proposal after which it gets aggressive and decreases the offer price geometrically. In response to that, the buyer agent keeps his offer steady. By the $13^{\text {th }}$ offer, the

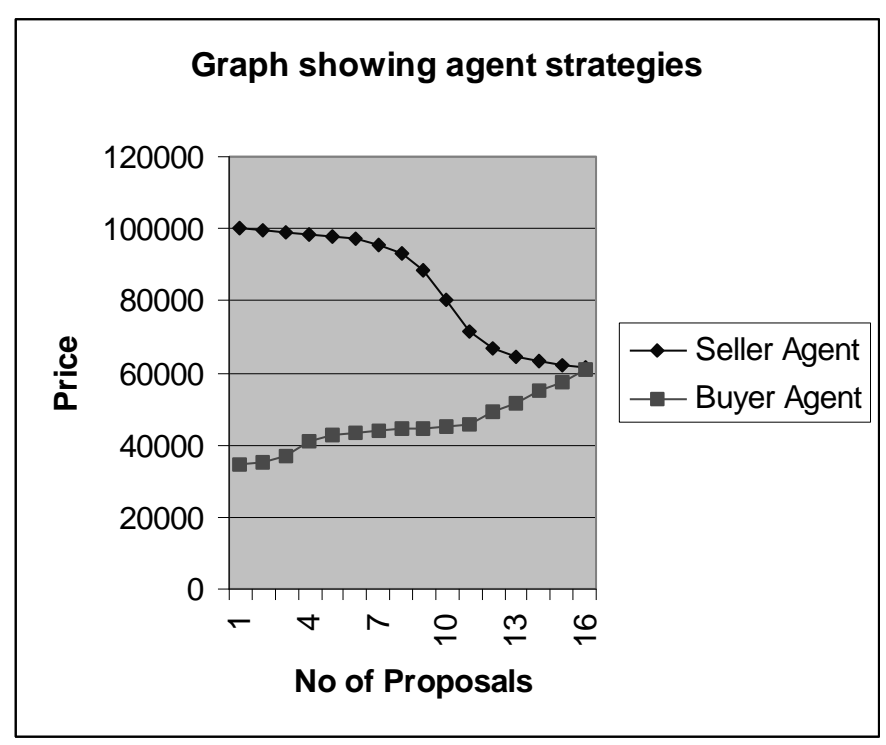

Fig. 7c: Graph showing negotiation pattern of agents 
seller starts loosing interest, going back to steady decreases. At this point, the buyer responds by getting aggressive, finally an agreement is reached on the $16^{\text {th }}$ proposal. The graph verifies the adaptive strategy adopted by the buyer agent.

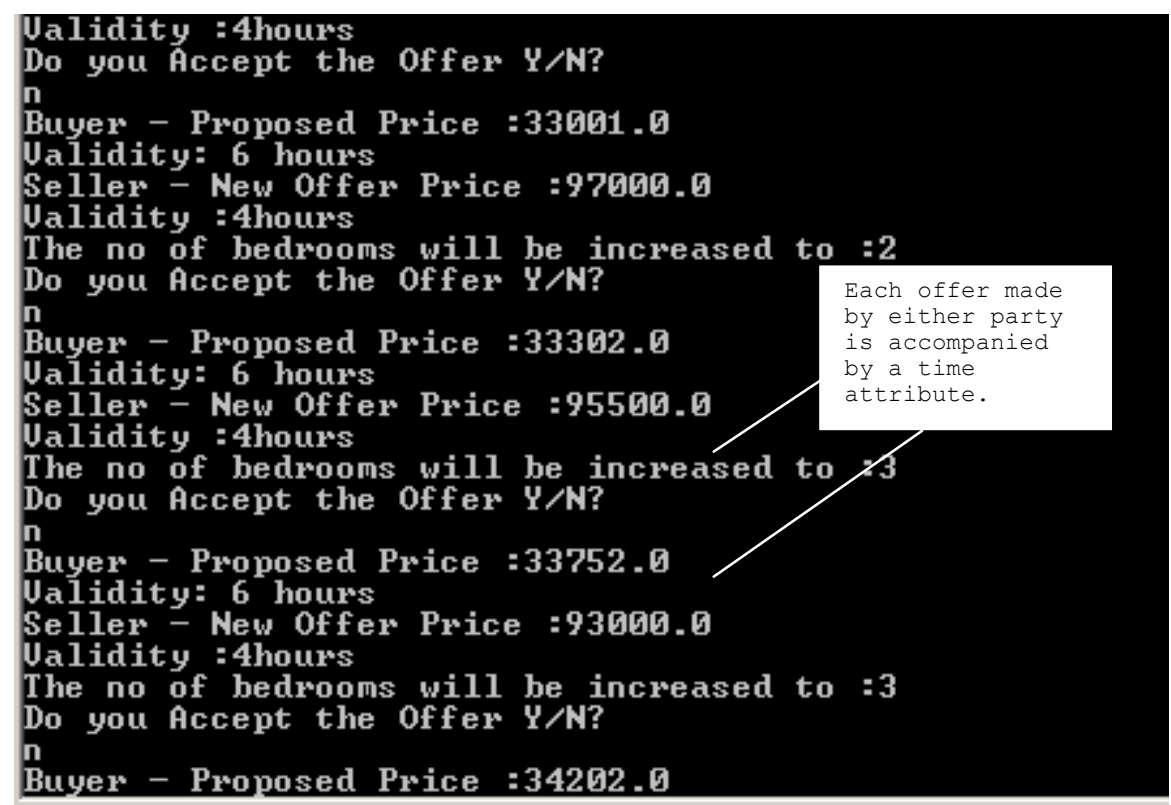

Fig. 7d: Time attribute functionality

\subsection{Time/Validity}

Figure $7 \mathrm{~d}$ above shows a negotiation scenario where each proposal made by either party is accompanied with a validity period, thus fulfilling the time attribute requirement of the framework.

\section{CONCLUSION}

SOLACE is a generic, multi-issue negotiation framework that meets today's electronic negotiation system needs. The framework recognizes the importance of strategies in negotiations and incorporates a time attribute in 
negotiation proposals. SOLACE can be adopted as a standard, serving as a model for future electronic negotiation system implementations. Thus, SOLACE would be used to characterize the nature and process of negotiations, formalize its aspects, and describe a wide range of possible structures and interactions. SOLACE, in compliance with current programming methodologies encourages component re-use and inheritance. Moreover, the adoption of a standard framework will make it easier to evaluate future electronic negotiation systems.

Our research involved the development of a proof of the concept Property Negotiation System, which can be easily enhanced for use at any Estate agency. The system is fully functional and can be used to automate the negotiation process of actual buying, selling, or renting houses. The same concept can be adopted in other multi-issue negotiation scenarios.

Another important characteristic of the framework is the involvement of humans at the agreement phase. In today's e-commerce transactions, humans designate agents to bargain on their behalf but still want to be involved before the final decisions are made. Existing negotiation systems overlook the importance of this, rendering them useful only to a handful of electronic commerce enthusiasts.

The PNS implemented is perhaps at the lower end of the scale in terms of the complexity involved in today's electronic negotiation systems. We are currently working on demonstrating the use of SOLACE in multi agent and multi-attribute scenarios with more complex strategies. Rigorous evaluation and testing will then be carried out to show that the framework can be used in both simple and complex developments.

We recognize that SOLACE—although robust and efficient—can still be improved upon. The framework can be expanded to incorporate current research areas in electronic negotiations such as ontology integration. Highly sophisticated agents, which can predict the opponent's strategy based on experience, can be used.

Finally, although SOLACE addresses many issues currently facing electronic negotiations, it is by no means a utopian solution. The dynamic nature of the e-commerce domain will make it impossible to find a single solution (framework) to 'all of the problems, all of the time' but rather to solve 'all of the problems, most of the time'. The latter is what the future 
enhancements to SOLACE will aim to achieve.

\section{REFERENCES}

http://interneg.org/inspire/doc/about_inspire.html. Accessed 12th December 2004.

http://wiki.cs.uiuc.edu/VisualWorks/What+are+frameworks\% Accessed 12th December 2004.

Bartolini, C., Preist, C., Jennings, N.R. 2002. A generic software framework for automated negotiation, AAMAS'02, Bologna, Italy.

Beam, C., Segev, A., Shantikumar, J.G. 1996. Electronic negotiation through internet-based auction, CITM Working Paper 96-WP-1019.

Benyoucef, M., Alj, H. and Keller, R.K. 2001. An Infrastructure for ruledriven negotiating software agents, $12^{\text {th }}$ International Workshop on Database and Expert Systems Applications, Munich, Germany

Bellosta, M., Brigui, I., Kornman, S. and Vanderpooten, D. 2004. A multicriteria model for electronic auctions, ACM Symposium on Applied Computing, Nicosia, Cyprus.

Bichler, M., Kalagnanam, J., Lee, H.S. and Lee, J. 2002. Winner determination algorithms for electronic auctions: a framework design, Proceedings of E-Commerce and Web Technologies: Third International Conference, EC-Web 2002, Aix-en-Provence, France.

Cardoso, H.L. and Oliveira, E. 2000. Using and evaluating adaptive agents for electronic commerce negotiations, IBERAMIA-SBIA 2000, 96-105.

Chavez, A. and Maes, P. 1996. KABASH: An agent marketplace for buying and selling goods. Proceedings of the 1st International Conference on the Practical Application of Intelligent Agents and Multi-Agent Technology, London, U.K., 75-90.

Jennings, N.R., Faratin, P., Lomuscio, A.R., Parsons, S., Sierra, C. and Wooldridge, M., 2001. Automated negotiation: prospects, methods and Challenges, International Journal of Group Decision and Negotiation, 10, 199-215.

Jennings, N.R. and Wooldridge, M.J. 1998. Applications of intelligent agents, In: Agent Technology: Foundations, Applications and Markets, edited by Jennings, N.R. and Wooldridge, M.J., Berlin, Germany, Springer-Verlag, $3-28$.

Johnson, R. 2004. http://st-www.cs.uiuc.edu/users/johnson/frameworks.html Accessed 30 ${ }^{\text {th }}$ December 2004.

Kersten, G.E., Noronha, S.J. and Teich, J. 2000. Are all E-commerce negotiations auctions? COOP'2000: Fourth International Conference on the Design of Cooperative Systems, Sopihia-Antipolis, France. 
Kersten, G. E. 2003. E-negotiations: towards engineering of technologybased social processes, InterNeg Research Paper INR 02/04.

Lee, K.J. 2000. Time-bounded framework for automated negotiation, International Conference on Advances in Infrastructure for Electronic Business, Science, and Education on the Internet

Rahwan, I., Ramchurn, S.D., Jennings, N.R., McBurey, P., Parsons, S. and Sonenberg, L. 2004. Argumentation-based negotiation, The Knowledge Engineering Review, 18, 343-375.

Ströbel, M. 2001a. Communication design for electronic negotiations, Proceedings of the $10^{\text {th }}$ World Wide Web Conference, Hong Kong, New York, N.Y., U.S.A., ACM Press, 9-20.

Strobel, M. 2001b. Design of Roles and Protocols of Electronic Negotiation, Electronic Commerce Research Journal, Special Issue on Market Design, 1, 335-353.

Tu, M.T., Seebode, C., Griffel, F. and Lamerdorf, W. 2001. DynamiCS: An Actor-based Framework for negotiating Mobile Agents, Electronic Commerce Research Journal 1, 101-117.

Wong, W.Y., Zhang, D.M. and Kara-Ali, M. 2000. Negotiating with experience, AAAI2000 Knowledge-Based Electronic Markets, Technical Report WS-00- 04, 85-90.

Zlatev, Z., Van Eck, P. 2003. An examination of the negotiation domain for electronic commerce information Proceedings of International Conference on Enterprise Information Systems. 